\title{
ESTUDO COMPARATIVO DA EFICÁCIA DE TRATAMENTOS PARA O MELASMA: KLIGMAN E CYSTEAMINE
}

\author{
COMPARATIVE STUDY OF THE EFFICACY OF TREATMENTS FOR \\ MELASMA: KLIGMAN AND CYSTEAMINE
}

Lara Dayane de Medeiros Leite ${ }^{1}$ Milena Nunes Alves de Sousa ${ }^{2}$ Livio e Vasconcelos do Egypto ${ }^{3}$

RESUMO: Objetivo: Analisar a eficácia de Kligman em comparação com Cysteamine para o tratamento de melasma em pacientes de 27 a 44 anos sem tratamento atualmente. Métodos: Trata-se de um ensaio clínico duplo cego. A pesquisa ocorreu na Clínica NeureDerm, localizada na cidade de Patos-PB. A amostra foi composta por 20 pacientes diagnosticados com melasma, no período de 2019 a 2020, que se adequavam aos critérios de inclusão e exclusão. Os dados da pesquisa foram obtidos através de um formulário elaborado e preenchido pelo pesquisador. A pesquisa seguiu respeitando os aspectos éticos presentes na Resolução no 466/2012, e 510/2016 do Ministério da Saúde. Resultados: A média de idade dos participantes foi de 33,5 anos, além disso, o fototipo mais acometido foi o $\mathrm{V}$, e que $45 \%$ dos pacientes já fizeram algum tratamento anterior. No que diz respeito à reposição hormonal, $45 \%$ dos pacientes relataram fazer uso de anticoncepcional oral. Em relação à exposição solar, $55 \%$ dos pacientes relataram se expor moderamente ao sol e $60 \%$ da amostra afirmaram aplicar protetor solar de 1 a 4 vezes ao dia, tendo uma média de 2,5 vezes. A amostra foi subdivida em 2 grupos que receberam as medicações Kligman $(n=10)$ e Cysteamine $(n=10)$, e a média de diminuição do MASI foi de 10,38 evidenciando melhora clínica global. A diferença entre a média do MASI inicial e final, para o grupo que usou Kligman foi de 10,62 e para o de Cysteamine foi 9,8. Conclusão: Ambos os tratamentos utilizados (Kligman e Cysteamine) são seguros e capazes de produzir melhora clínica, porém, quando comparados, observa-se uma pequena vantagem porcentual de melhora para o Kligman. Os autores sugerem estudos adicionais, comparando as duas drogas em uma amostra maior, assim como pesquisas com outras drogas.

\footnotetext{
${ }_{1}^{1}$ Graduanda em Medicina pelo Centro Universitário de Patos (UNIFIP).

${ }^{2}$ Drª e Pós-Doutora em Promoção de Saúde, Pós-Doutora em Sistemas Agroindustriais, Docente no Curso de Medicina do UNIFIP.

${ }^{3}$ Esp. em Dermatologia, Docente no Curso de Medicina do UNIFIP.
} 
Palavras chave: Melasma. Kligman. Cysteamine. Tratamento. Dermatologia.

ABSTRACT: Objective: To analyze the efficiency of Klingman in comparison to Cysteamine, for the melasma treatment in patients from 27 to 44 years, without a current treatment. Methods: This was a blind clinical double trial. The research happened at NeureDerm clinic, located in Patos city, PB. The sample comprised 20 patients diagnosed with melasma in the period from 2019 to 2020, which fitted in the criteria of inclusion and exclusion. The datas of the research were obtained through an elaborated form and completed by the researcher. The research respected the ethics apects present in the Resolution number 466/2012 and 510/2016 of the Health Ministry. Results: The average age of the participants was 33,4 years old, furthermore, the more affected phototype was the $V$ and that $45 \%$ of the patients had done some treatment before. Concerning to the hormonal reposition, $45 \%$ of the patients said that they used oral contraceptive pills. In relation to the sunlight exposure, $55 \%$ of the patients said that they exposured themselves moderately, and $60 \%$ of the sample said that they used the sunscreen from 1 to 4 times per day, with an average of 2,5 times. The sample was splited in two groups that received the medicine Kligman $(n=10)$ and Cysteamine $(n=10)$, and the MASI average of decreasing was 10,38, highlighting the overall clinical improvement. The difference between the initial and the final MASI rates for the group using Kligman was for 10,62 and for the group using Cysteamine was of 9,8. Conclusion: Both treatments (Kligman and Cysteamine) are safe and able to produce clinical improvement, but, when we compare they both, we can see a little advantage percentage of improvement in Klingman. The aurhors suggest additional studies, comparing the two medicines, in a bigger sample, as well as researches with other drugs.

Keywords: Melasma. Kligman. Cysteamine. Treatment. Dermatology. 


\section{INTRODUÇÃO}

O melasma, também conhecido como cloasma, é um distúrbio hiperpigmentar benigno da pele, em que a epiderme do local apresenta uma hiperatividade melanocítica com elevado número de melanossomas com alto grau de maturação, que promovem hiperpigmentação em todas as camadas epidérmicas (SALLES; REIS; SOUZA, 2018; MENON et al., 2019; RAJANALA; MAYMONE; VASHI, 2019; GHELLERE; BRANDÃO, 2020). Para Menon et al. (2019), são muitos os fatores de risco associados, contudo, a patogênese exata ainda é uma incógnita.

Afeta pessoas com tipos de pele mais escura (tipos III e IV), ambos os sexos, e abrange todas as raças, entretanto, é mais prevalente em mulheres em idade fértil, gestantes, mais comuns em hispânicos, pacientes de pele escura e asiáticos. Os fatores de risco mais comuns são exposição excessiva ao sol sem uso de protetor solar, utilização de anticoncepcionais, pré-disposição genética e gravidez. Todos os tipos de pele estão propícios ao desenvolvimento dos distúrbios pigmentares, porém, em pacientes com pele mais escura a terapia geralmente é dificultada, exigindo aplicação tópica de agentes despigmentantes como a hidroquinona, prevenção e proteção solar e às vezes, aplicações de peelings químicos, com o intuito de reduzir a hiperpigmentação, tomando certo cuidado no clareamento indesejado da pele normal (AVRAM et al., 2008; BECKER et al., 2017; SARKAR et al;. 2019).

Já foi comprovado que cosméticos, esteroides e fotossensibilizantes podem gerar melasma. As drogas ficam ligadas aos pigmentos da melanina da pele, no olho e na orelha, causando pigmentação, como também há predisposição individual, que é a maior concentração de feomelanina na pele (ELVIRO JUNIOR et al., 2018).

O melasma não provoca repercussões clínicas graves, contudo, distúrbios como esse, incomodam e afetam a autoestima do paciente, o que provoca em um terço desses pacientes, grande impacto estético crônico e significativo, principalmente para as mulheres, de modo a promover um severo sofrimento psicossocial e emocional, interferindo em sua qualidade de vida (URASAKI; 
MANDELBAUM; GONÇALVES, 2013; OLIVEIRA et al., 2019). Além disso, a terapia utilizada pode ser frustrante, gerando uma preocupação para os médicos, devido à dificuldade no clareamento das manchas com aplicações de diversos ativos dermatológicos e métodos, resultando apenas uma pequena melhora (AVRAM et al., 2008).

A abordagem terapêutica não deve abranger somente o distúrbio orgânico (URASAKI et al., 2018). Por esse motivo, o paciente deve ser avaliado como um todo, levando em consideração a história genética, uso de terapias hormonais, exposição solar, comorbidades e o impacto da doença na sua vida social. O paciente deve estar esclarecido sobre a doença em si e sobre os fatores que tendem a piorar o quadro, isto e, o uso de drogas como anticoncepcionais, terapias de reposição hormonal e cosméticos fototóxicos. Deve-se instituir o uso do protetor solar, visto que é indispensável e de extrema importância durante o tratamento (HEXSEL et al., 2015; URASAKI et al., 2018).

O tratamento do melasma tem por objetivo prevenir ou reduzir a gravidade de sua recorrência, clareamento em tempo reduzido, melhora do seu aspecto cosmético e redução da área afetada. Os métodos utilizados atualmente são os métodos químicos, que incluem hidroquinona, corticosteroides, retinoides, ácido ascórbico, antioxidantes orais; métodos físicos que abrangem a microdermoabrasão, peelings químicos (ácido retinoico, ácido glicólico, ácido salicílico), uso de lasers e luz intensa pulsada (TRIVEDI; YANG; CHO, 2017; ARROWITZ et al, 2019; CUNHA; SILVA; OLIVEIRA, 2020; GHELLERE; BRANDÃO, 2020; MCKESEY; TOVAR-GARZA; PANDYA, 2020).

No presente estudo será testada a eficácia da fórmula de Kligman comparada à fórmula Cysteamine. A fórmula de Kligman, atualmente, mais utilizada em tratamento de melasma, é uma combinação de ácido retinóico, hidroquinona e corticoide, com o objetivo de potencializar o efeito clareador da hidroquinona de modo isolado; a Cysteamine é uma droga relativamente nova, que tem função antioxidante superpotente, inibindo tirosinase e peroxidase, sequestrando dopaquinona e atuando diretamente na origem do melasma.

Essa discussão torna-se oportuna, pois, a temática da eficácia de seus tratamentos ainda é pouco abordada, e vem despertando, no meio científico, vários 
questionamentos, dentre eles: qual a eficácia da Kligman em comparação com a Cysteamine para o tratamento do melasma em pacientes de 27 a 44 anos sem tratamento atualmente?

Portanto, a investigação objetiva analisar a eficácia da Kligman em comparação com a Cysteamine para o tratamento do melasma em pacientes de 27 a 44 anos sem tratamento atualmente. Assim sendo, o presente estudo confere relevância significativa no âmbito da promoção da saúde, enfatizando a melhor alternativa para os casos de melasma, visto que ainda há muito a desmistificar acerca do objeto de estudo.

\section{MATERIAL E MÉTODOS}

O presente estudo é definido por ensaio clínico duplo cego, que tem por descrição um método de ensaio clínico realizado com seres humanos onde nem o examinado nem o examinador sabem o que está sendo utilizado como variável em um espaço de tempo. É regularmente utilizado como critério de validação de práticas experimentais quantitativas em ciência. Segundo Pronadov e Freitas (2013), esse tipo de pesquisa objetiva gerar conhecimentos para aplicação prática, dirigidos à solução de problemas específicos.

Foi desenvolvido na Clínica NeureDerm, localizada na Rua Bossuet Wanderley, 411, CEP: 58.000-410, no bairro Brasília, Patos, Paraíba-PB. A pesquisa foi realizada no período de Outubro de 2019 a Abril de 2020. A amostra não probabilística por conveniência foi constituída por 20 pacientes que foram atendidos entre 2019 e 2020.

Os pacientes foram contactados via telefone e ao manifestarem interesse em participar do estudo, foi verificado se os mesmos atendiam aos critérios de elegibilidade pré-determinados. A amostragem foi obtida mediante análise do perfil dos pacientes que foram selecionados adotando-se os seguintes critérios de inclusão: (1) pacientes com idade entre 27 e 44 anos; (2) não estar em tratamento 
ativo; (3) apresentar melasma com confirmação diagnóstica pelos pesquisadores; (4) não apresentar intolerância ou alergia às medicações propostas.

Portanto, após a pesquisa ser aprovada pelo Comitê de Ética em Pesquisa (CEP) do Centro Universitário de Patos (UNIFIP), cujo número do parecer de aprovação 3.646.934, foram coletados os dados a partir de prontuários criados daqueles pacientes que atenderam aos critérios de inclusão através do formulário previamente elaborado.

Os dados foram coletados em um formulário elaborado e preenchido pelo pesquisador, contento as seguintes variáveis: idade, sexo, motivo da consulta, sinais e sintomas no momento do atendimento, patologias concomitantes, fatores de risco, história de tratamento anterior, características da pele, cuidados diários, localização da mancha, diagnóstico clínico, tratamento proposto. Além disso, foi utilizado o Índice de Área e Gravidade de Melasma (MASI), que teve com exatidão o grau de acometimento do melasma.

Os grupos foram divididos de modo aleatório, sigiloso e por sorteio, com duplo cegamento, conforme características semelhantes para ambos os grupos. A aleatorização evitou fatores de confundimento e viés no estudo. Logo, os grupos foram subdivididos em A e B, com 10 pacientes em cada, em que se aplicou o tratamento com Kligman e Cysteamine, respectivamente. Comparando seus fatores de eficácia através de melhora clínica e fotográfica do melasma.

O tratamento contou com o uso de duas formulações preconizadas, Kligman e Cysteamine, sendo Kligman composto por ácido retinóico 0,05\%, hidroquinona 5\%, fluocinolona acetonida $0,05 \%$ e gel creme, e Cysteamine composto por Cisteamina $5 \%$, base despigmentante Roval (neutralizador de odores $3 \%$, óleo de cravo $1,5 \%$, thalasferas de vitamina C $1 \%$, vitamina A $0,5 \%$, qsp gel e ácido resistente). O (a) paciente fez uso das 19:00 às 22:00, todos os dias, durante 04 meses. Após retirada, realizou higienização facial com o sabonete adequado. Além disso, foi imprescindível o uso do protetor solar FPS 50, que foi aplicado 03 vezes ao dia (7:00, 11:00 e 15:00). Foi proibido o uso de qualquer outra substância na pele, dispensado apenas o uso de maquiagem. Para acompanhar a evolução do tratamento, foi utilizado o método de avaliação fotográfica inicial e ao finalizar o tratamento. Caso algum participante não conseguisse seguir estritamente o 
protocolo proposto, o mesmo seria excluído da pesquisa. Também seriam excluídos os participantes que apresentassem efeitos adversos à medicação.

A análise dos dados provenientes do ensaio clínico duplo cego foi através do Índice de Área e Gravidade de Melasma (MAIS), o qual é a medida utilizada para fornecer uma quantificação mais precisa da gravidade e das alterações durante 0 tratamento do melasma. O escore MASI é calculado por avaliação subjetiva de 3 fatores: área de envolvimento $(A)$, escuridão $(D)$ e homogeneidade $(H)$, com a testa (f), região malar direita $(\mathrm{rm})$, região malar esquerda $(\mathrm{Im})$ e queixo (c), correspondendo a 30\%, 30\%, 30\% e 10\% da face total, respectivamente. A área de envolvimento em cada uma dessas 4 áreas recebe um valor numérico de 0 a $6(0=$ sem envolvimento; 1: < 10\%; 2: 10\% - 29\%; 3: 30\% - 49\%; 4: 50\% - 69\%; 5: 70\% $89 \%$ e 6: $90 \%$ - 100\%). A escuridão e a homogeneidade são classificadas em uma escala de 0 a 4 (0: ausente; 1: leve; 2: suave; 3: marcado; e 4: máximo). O escore MASI é calculado adicionando a soma das classificações de gravidade para escuridão e homogeneidade, multiplicada pelo valor da área de envolvimento, para cada uma das 4 áreas faciais. A fórmula utilizada é:

$M A S I=0,3 A(D+H)+0,3 A(D+H)+0,3 A(D+H)+0,1 A(D+H)$.

Em que foi aplicada para cada região do rosto (queixo - c, região malar esquerda - Im, testa - $f$, região malar direita - rm). Foi avaliado e comparado de acordo com MASI inicial e MASI final, sendo verificado se houve diminuição do valor final ou não, com melhora ou piora, respectivamente. Posteriormente, seguiu-se para análise estatística.

\section{RESULTADOS}

Observou-se que a idade dos participantes do estudo variou de 27 a 44 anos, com média de 33,5 anos. Dos 20 pacientes incluídos, 95\% $(n=19)$ eram mulheres e 
$5 \%(\mathrm{n}=1)$ homem. Além disso, as idades mais comuns foram 27 anos, 31 anos, 34 anos, 36 anos, 37 anos e 38 anos, todas com $10 \%(n=2)$, cada.

A tabela 1 mostra que $30 \%(n=6)$ dos pacientes tinham fototipo tipo $V$, seguido do tipo IV, com $25 \%$ ( $n=5)$. Após a avaliação, percebeu-se que o tempo de surgimento do Melasma variou de 1 a 17 anos, com média de 7,55 anos (aproximadamente 90,6 meses). No mais, foi evidenciado que $40 \%$ dos pacientes $(n=8)$ possuíam melasma entre 1 e 5 anos ou entre 6 e 10 anos.

Tabela 1: Perfil epidemiológico dos pacientes participantes do estudo - fototipo e tempo do melasma $(n=20)$.

\begin{tabular}{ccc}
\hline Fototipo & $\mathbf{n}$ & $\%$ \\
\hline I & 1 & 5 \\
II & 4 & 20 \\
III & 4 & 20 \\
IV & 5 & 25 \\
V & 6 & 30 \\
\hline Tempo de Melasma & $\mathbf{n}$ & $\%$ \\
\hline $1-5$ anos & 8 & 40 \\
$6-10$ anos & 8 & 40 \\
Acima de 10 anos & 4 & 20 \\
\hline Total & $\mathbf{2 0}$ & $\mathbf{1 0 0}$
\end{tabular}

Fonte: Dados de Pesquisa 2019/2020.

De acordo com a tabela 2, 45\% ( $n=9)$ dos pacientes fizeram algum tratamento anterior e 10\% ( $n=2)$ possuíam uma patologia dermatológica associada (seboríase e queloide). Dos 20 pacientes analisados, 45\% (n=9) faziam uso de reposição hormonal com anticoncepcional oral (com a média de 9,3 meses, sendo o mínimo 2 meses e o máximo 4 anos de uso), e 60\% ( $n=12)$ faziam uso da proteção solar, cuja aplicação variou de 1 a 4 vezes ao dia, sendo a média de 2,5 vezes. 
Tabela 2: Perfil epidemiológico dos pacientes participantes do estudo - tratamento anterior, patologia dermatológica associada, reposição hormonal, uso de protetor $\operatorname{solar}(n=20)$.

\begin{tabular}{lcc}
\hline Tratamento anterior & $\mathbf{n}$ & $\%$ \\
\hline Sim & 9 & 45 \\
Não & 11 & 55 \\
\hline Patologia dermatológica associada & $\mathbf{n}$ & $\%$ \\
\hline Sim & 2 & 10 \\
Não & 18 & 90 \\
\hline Reposição hormonal & $\mathbf{n}$ & $\%$ \\
\hline Sim & 9 & 45 \\
Não & 11 & 55 \\
\hline Uso de protetor solar & $\mathbf{n}$ & $\%$ \\
\hline Sim & 2 & 10 \\
Não & 18 & 90 \\
\hline Total & $\mathbf{2 0}$ & $\mathbf{1 0 0}$ \\
\hline
\end{tabular}

Fonte: Dados de Pesquisa 2019/2020.

Segundo a tabela 3, 55\% $(n=11)$ dos pacientes estudados ficavam expostos ao sol moderadamente, seguido de $25 \%(n=5)$, cuja exposição era esporádica.

Tabela 3 - Perfil epidemiológico dos pacientes participantes - exposição solar $(n=20)$.

\begin{tabular}{ccc}
\hline Fototipo & $\mathbf{n}$ & $\%$ \\
\hline Leve & Leve & 4 \\
Esporádica & Esporádica & 5 \\
Moderada & Moderada & 11 \\
Total & $\mathbf{2 0}$ & $\mathbf{1 0 0}$
\end{tabular}

Fonte: Dados de Pesquisa 2019/2020.

Em $50 \%$ dos pacientes $(n=10)$, foi utilizada a droga Cysteamine, e nos outros $50 \%(n=10)$, foi utilizado a droga Kligman. Dos 10 pacientes que fizeram uso de Kligman, 9 (90\%) obtiveram resultado positivo e 1 (10\%) obteve resultado negativo. Já com a droga Cysteamine, 10 pacientes iniciaram o tratamento, porém 3 participantes (15\%) não completaram o estudo (perda do seguimento ou gestação), sendo assim, os 7 pacientes válidos obtiveram resultado positivo. Como mostra a 
tabela $4,80 \%$ dos pacientes $(n=16)$ obtiveram resultado positivo e apenas $5 \%(n=1)$ obteve resultado negativo. O MASli teve uma média de 15,65 pontos, com desvio padrão de 8,93 pontos, o MASIf teve uma média de 5,27 pontos, com desvio padrão de 4,88 pontos. A diferença média nos escores MAIS, antes e após o tratamento, foi de 10,38 pontos, com máximo de 30,8 e o mínimo de 5,9, ou seja, houve aumento de 5,9 pontos em um dos escores no final do tratamento, evidenciando piora. De acordo com os resultados, os escores médios da escala MAIS, no início do tratamento, foram mais elevados entre pacientes com fototipos IV e II, exposição solar moderada e sem relação com o uso de anticoncepcional oral.

Poucos efeitos adversos foram observados, como eritema e edema leves transitórios no pós-peeling imediato.

Entretanto, o valor de Qui-quadrado, nesse estudo, foi de 0,388, isto é, essas diferenças não foram estatisticamente significativas (valores de $p<0,05$ ), podendo então, ser prescrita as duas medicações, pois ambas são cientificamente comprovadas como eficazes para o tratamento do melasma. Contudo, comparando os MASI separadamente, observou-se que a droga Kligman obteve uma porcentagem maior de melhora (71\%), em relação ao Cysteamine (55\% de melhora).

Tabela 4 - Resultados MASli e MASIf $(n=17)$.

\begin{tabular}{|c|c|c|c|c|c|}
\hline MAIS & \multicolumn{3}{|c|}{$\mathbf{n}$} & \multicolumn{2}{|c|}{$\%$} \\
\hline Positivo & \multicolumn{3}{|c|}{16} & \multicolumn{2}{|c|}{80} \\
\hline Negativo & \multicolumn{3}{|c|}{1} & \multicolumn{2}{|c|}{5} \\
\hline Omisso & \multicolumn{3}{|c|}{3} & \multicolumn{2}{|c|}{15} \\
\hline MASli e MASIf & $\mathbf{N}$ & & Média & \multicolumn{2}{|c|}{ Erro desvio } \\
\hline MASli & 20 & & 15,6500 & \multirow{3}{*}{\multicolumn{2}{|c|}{$\begin{array}{l}8,93099 \\
4,88988\end{array}$}} \\
\hline MASIf & 17 & & 5,2706 & & \\
\hline \multicolumn{3}{|c|}{$\mathrm{N}$ válido } & & & \\
\hline \multicolumn{2}{|c|}{ Qui-Quadrado } & Valor & $\begin{array}{l}\text { Significância } \\
\text { assintótica } \\
\text { (bilateral) }\end{array}$ & $\begin{array}{l}\text { Sig exata } \\
\text { (2 lados) }\end{array}$ & $\begin{array}{l}\text { Sig exata } \\
\text { (1 lado) }\end{array}$ \\
\hline \multirow{6}{*}{\multicolumn{2}{|c|}{$\begin{array}{l}\text { Qui-Quadrado de Pearson } \\
\text { Correção de continuidade }{ }^{\mathrm{b}} \\
\text { Razão de verossimilhança } \\
\text { Teste exato de Fisher } \\
\text { Associação Linear por Linear } \\
\mathrm{N} \text { de casos válidos }\end{array}$}} & 0,744 & 0,388 & \multirow{6}{*}{1,000} & \multirow{6}{*}{0,588} \\
\hline & & 0,000 & 1,000 & & \\
\hline & & 1,105 & 0,293 & & \\
\hline & & & & & \\
\hline & & 0,700 & 0,403 & & \\
\hline & & 17 & & & \\
\hline
\end{tabular}

Fonte: Dados de Pesquisa 2019/2020. 


\section{DISCUSSÃO}

O melasma ocorre com mais frequência em pessoas com a pele mais escura, em idade reprodutiva. A idade média do surgimento na população brasileira se dá a partir dos 30 anos (MIOT et al., 2007), mas, pesquisa indicou média de idade 25,80 $( \pm 8,69)$ anos (OLIVEIRA et al., 2019) e, na presente investigação, a população apresentou medida de idade de 27 anos, apontando esse marco para o surgimento do melasma. Pode-se então estimar, uma média variando entre 27 e os 30 anos de idade.

O diagnóstico é clínico, podendo utilizar alguns recursos como auxílio, são eles: luz de Wood, em que é realizado em ambiente escuro e classifica o melasma em quatro tipos, de acordo com a profundidade do pigmento (MIOT et al., 2009), além dele, existe também a dermatoscopia, realizada com equipamento óptico que permite aproximação de até 400 vezes, evidenciando melanina no local (MEDEIROS et al., 2016). O uso desses métodos, é de extrema importância no diagnóstico, visto que vão auxiliar na classificação, escolha do método de tratamento e acompanhamento (MIOT et al., 2009).

Em relação ao fototipo, houve predomínio do V (30\%), nesta pesquisa. Esse achado apresenta certa correlação com a literatura, uma vez que aponta como os tipos mais melanizados, segundo a classificação de Fitzpatrick, o III, IV e V (HANDEL, 2013).

Sabe-se que o melasma é mais comum em mulheres na idade fértil e que o sexo masculino representa apenas $10 \%$ dos casos (MIOT, 2009; OLIVEIRA et al., 2019), dado análogo à amostra deste estudo, em que, 95\% foram mulheres e 5\% homem.

Quanto ao uso de anticoncepcional oral, Handel (2013) afirma que entre $8 \%$ e $34 \%$ das mulheres que utilizam desenvolvem a doença e muitas referem que o surgimento do melasma se deu após o início desse uso. Em estudo na Bahia, dentre as mulheres participantes, $80 \%$ delas relataram fazer uso de anticoncepcional 
(OLIVEIRA et al., 2019). Nesta investigação, 47,36\% das pacientes relataram reposição hormonal com anticoncepcional oral. Além disso, dos pacientes avaliados, apenas 10\% tinha alguma doença sistêmica associada (seboríase e queloide), no entanto, nenhuma das patologias citadas prejudica o tratamento proposto para melasma.

Em relação à fotoproteção, Purim e Avelar (2012), Oliveira et al. (2019), Paula, Moraes e Oliveira (2019), Cunha, Silva e Oliveira (2020), Ghellere e Brandão (2020) afirmam que, atualmente, o uso de protetor solar é considerado um redutor da intensidade do melasma, pois ajuda a estabilizar os benefícios obtidos com o tratamento aqui descrito. Apesar da assertiva, parece que a continuidade do uso não fez tanta diferença para esta pesquisa, já que um dos achados importantes foi o fato de que $60 \%$ da amostra estudada usava regularmente o protetor solar, com quase $34 \%$ com aplicação do produto três vezes ao dia.

Diante do resultado, vale ressaltar acerca da forte incidência de radiação Ultravioleta (UV) durante todo o ano (TAVARES; SOUSA; CARVALHO, 2020), seguido do clima local, demasiadamente quente, associado aos costumes locais, em que a população toma poucos cuidados com a saúde da pele, portanto, a incidência de melasma é frequentemente maior.

O Kligman tem como base a hidroquinona, utilizada há mais de cinco décadas para tratamento de melasma, a qual atua bloqueando a conversão de dopa em melanina, através da enzima tirosinase. Além disso, conta com a associação do ácido retinóico, que reduz a hiperpigmentação pela indução de descamação da pele. A sua combinação com a hidroquinona gera uma correção da pigmentação por correção do fotodano. O Cystemine é um radioprotetor que fornece proteção contra as consequências mutagênicas e letais da radiação ionizante (FARSHI; MANSOURI; KASRAEE, 2018), sobretudo, possui função de antioxidante superpotente, inibindo tirosinase e peroxidase e sequestrando dopaquinona, atuando diretamente na origem do melasma (STEINER et al., 2019).

É sabido que os tratamentos, apesar de apresentarem bons resultados, podem gerar efeitos colaterais, entre os quais está o desencadeamento de um processo inflamatório, que inclui dermatite de contato alérgica ou irritativa, hiperpigmentação pós-inflamatória (FIGUEIRÓ; FIGUEIRÓ FILHO; COELHO, 2008), 
além de prurido e ardência. Todos os efeitos que surgiram após uso dos ácidos foram transitórios e leves, como eritema e ardor, mas não houve necessidade de interrupção de nenhuma das duas terapêuticas em decorrência dos efeitos adversos.

A meta terapêutica, como citada anteriormente, é a redução significativa ou total da mancha, a qual as pessoas portadoras de melasma geralmente têm certo receio de sair sem cobri-las, pois, se sabe que a aparência está ligada à autoimagem e autoestima, sendo assim, a pele funciona como um importante órgão de comunicação social e a sua visibilidade não íntegra, pode estigmatizar e reprimir as relações psicossociais (MEDEIROS et al., 2016). "As dermatoses podem afetar a autoestima e contribuem para causar sentimentos que pode se manifestar como ansiedade, tristeza ou até depressão" (OLIVEIRA et al., 2019, p. 440). Assim, com a melhora da aparência da pele, supõem-se melhorias na autoestima $e$, consequentemente, no nível de qualidade de vida do grupo.

Por fim, é vasta a literatura sobre as várias opções para tratamento de controle do melasma, porém, se questiona a falta de estudos bem controlados e reprodutíveis sobre o assunto (TRIVEDI; YANG; CHO, 2017; ARROWITZ et al., 2019; AUSTIN; NGUYEN; JAGDEO, 2019; BAGATIN; HASSUN; TALARICO, 2019 MENON et al., 2019; WANG; JHAWAR; SAEDI, 2019; CUNHA; SILVA; OLIVEIRA, 2020; GHELLERE; BRANDÃO, 2020; MCKESEY; TOVAR-GARZA; PANDYA, 2020). Por mais que exista diversas opções, não há estudos suficientes que comparem e comprovem a eficácia dos mesmos, ou seja, a temática da eficácia de seus tratamentos ainda é pouco abordada e vem despertando, no meio científico, vários questionamentos.

Deve atentar para o método utilizado na mensuração do evento final de interesse se foi o duplo cego, ou seja, nem o paciente, nem o profissional que está fazendo a avaliação sabem qual tratamento foi aplicado. O médico pode, inconscientemente, não valorizar as queixas das pacientes que pertencem ao grupo do tratamento, falseando os resultados. O próprio paciente pode ser simpático a um determinado tipo de tratamento e não informar ao médico todas as queixas que tem. O método duplo-cego tenta eliminar este potencial, tipo de erro e qualquer tipo de viés que possa vir a existir (OLIVEIRA; PARENTE, 2010). 


\section{CONCLUSÃO}

De acordo com os resultados obtidos ao final do estudo, conclui-se, portanto, que ambos os tratamentos utilizados (Kligman e Cysteamine) são seguros e capazes de produzir melhora clínica, porém, quando se compara os dois, observa-se uma pequena vantagem porcentual de melhora para o Kligman. No mais, a média de MASI no início do tratamento foi mais elevada em pacientes com exposição solar moderada, seguido de fototipo IV e que faziam uso de anticoncepcional oral.

Os autores sugerem estudos adicionais, comparando as duas drogas em uma amostra maior, assim como pesquisas com outras drogas.

\section{REFERÊNCIAS BIBLIOGRÁFICAS}

ARROWITZ, C. et al. Effective Tyrosinase Inhibition by Thiamidol Results in Significant Improvement of Mild to Moderate Melasma. J Invest Dermatol., v. 139, n. 8, p. 1691-8, 2019.

AUSTIN, E.; NGUYEN, J. K.; JAGDEO, J. Topical Treatments for Melasma: A Systematic Review of Randomized Controlled Trials. J Drugs Dermatol., v. 18, n. 11, 2019.

AVRAM, M. R. et al. Atlas colorido de Dermatologia Estética. Rio de Janeiro: McGraw-Hill Interamericana do Brasil, 2008.

BAGATIN, E.; HASSUN, K.; TALARICO, S. Revisão sistemática sobre peelings. Surgical \& Cosmetic Dermatology, São Paulo, v. 1, n. 1, p. 37-46, 2019.

BECKER, S. et al. Melasma: Ein Update zu Klinik, Therapie und Prävention. Hautarzt, v. 68, n. 2, p. 120-126, 2017.

CUNHA, I. G.; SILVA, C. P.; OLIVEIRA, G. B. B. Principais tratamentos do melasma. Humanidades e tecnologia (FINOM), v. 1, n. 23, p. 302-315, 2020.

ELVIRO JUNIOR, M. et al. Melasma. In: ELVIRO JUNIOR, M. et al. Ambulatório de dermatologia em APS. São Paulo: Martinari, 2018. Cap. 6, p.107-110.

FARSHI, S.; MANSOURI, P.; KASRAEE, B. Efficacy of cysteamine cream in the treatment of epidermal melasma, evaluating by Dermacatch as a new measurement method: a randomized double blind placebo controlled study. Journal of Dermatological Treatment, v. 29, n. 2, p. 182-189, 2018.

FIGUEIRÓ, T. L. M.; FIGUEIRÓ FILHO, E. A.; COELHO, L. R. Pele e gestação: aspectos atuais 
dos tratamentos e drogas comumente utilizados. Femina, v. 36, n. 8, p. 511-521, 2008.

GHELLERE, I. C.; BRANDÃO, B. J. F. A pele e o melasma. BWS Journal, v. 3, p. 1-11, 2020.

HANDEL, A. C. Fatores de risco para melasma facial em mulheres: um estudo casocontrole. 2013. 100 fls. Dissertação (Mestrado em Patologia) - Universidade Estadual Paulista, Botucatu, 2013.

HEXSEL, D. et al. Epidemiology of melasma in Brazilian patients: a multicenter study. Int $\mathbf{J}$ Dermatol, v. 53, n. 4, p. 440-4, 2014.

MCKESEY, J., TOVAR-GARZA, A.; PANDYA, A. G. Melasma Treatment: An Evidence-Based Review. Am J Clin Dermatol., v. 21, n. 2, p. 173-225, 2020.

MEDEIROS, J. K. G. et al. Combinação terapêutica no tratamento do melasma. CuidArte, Enferm, v. 10, n. 2, p. 180-187, 2016.

MENDONÇA, P. R. Protocolos estéticos para o melasma. Revista Saúde Integrada, v. 12, n. 25, p. 36, 2019.

MENON, A. et al. A Split Face Comparative Study of Safety and Efficacy of Microneedling with Tranexamic Acid versus Microneedling with Vitamin $C$ in the Treatment of Melasma. Indian Dermatol Online J., v, 11, n. 1, p. 41-5, 2019.

MIOT, L. D. B. et al. Estudo comparativo morfofuncional de melanócitos em lesões de melasma. An. Bras. Dermatol. Rio de Janeiro, v. 82, n. 6, p. 529-534, dez. 2007.

MIOT, L. D. B. et al. Fisiopatologia do melasma. An. Bras. Dermatol., Rio de Janeiro, v. 84, n. 6, p. 623-635, dez. 2009.

PAULA, V. G.; MORAES, D. N.; OLIVEIRA, A. S. Contribuições do farmacêutico defronte a atenuação do melasma durante e depois da gestação. Revista JRG de Estudos Acadêmicos, v. 2, n. 4, p. 220-235, 2019.

PURIM, K. S. M.; AVELAR, M. F. S. Fotoproteção, melasma e qualidade de vida em gestantes. Revista Brasileira de Ginecologia e Obstetrícia, v. 34, n. 5, p. 228-234, 2012.

OLIVEIRA, A. A. et al. Impacto do Melasma na Autoestima de Mulheres. Id on Line Rev. Mult. Psic., v.13, n. 48m p. 435-443, 2019.

OLIVEIRA, M. A; PARENTE, R. C. Entendendo ensaios clínicos randomizados. Brazilian Journal of Videoendoscopic Surgery, [s. I.], v. 3, n. 4, p. 176-180, 2010.

RAJANALA. S.; MAYMONE, M. B. C.; VASHI, NA. Melasma pathogenesis: a review of the latest research, pathological findings, and investigational therapies. Dermatol Online J., v. 25, n. 10 , p. 13030, 2019.

SALLES, F. A.; REIS, R. C.; SOUZA, M. D. Fisiopatologia do melasma e tratamentos: Uma Causa Possível. Rev Con Eletron, v. 15, n. 1, p. 1866-74, 2018.

SARKAR, R. et al. Clinical and epidemiologic features of melasma: a multicentric cross-sectional study from India. Int J Dermatol., v. 58, n. 11, p. 1305-10, 2019

STEINER, D. et al. Treatment of melasma: systematic review. Surgical \& Cosmetic Dermatology, v. 1, n. 2, p. 87-94, 15 mar. 2009.

TAVARES, D. S.; SOUSA, M. N. A.; CARVALHO, F. K. Perfil epidemiológico de pacientes oncológicos em um serviço secundarista. Revista Brasileira de Educação e Saúde, Pombal, 
v. 10, n. 1, p. $122-128,2020$.

TRIVEDI, M. K.; YANG, F. C.; CHO, B. K. A review of laser and light therapy in melasma. Int J Womens Dermatol., v. 3, n. 1, p. 11-20, 2017.

URASAK, M. B. M. Conhecimento, atitude e prática da equipe de saúde sobre melasma na gravidez. Av Enferm., v. 36, n. 1, p. 40-9, 2018.

URASAK, M. B. M.; MANDELBAUM, M. H. S.; GONÇALVES, R. Impactos psicossociais associados às manchas gravídicas. Cogitare Enferm., v. 18, n. 4, p. 655-62, 2013,

WANG, J. V.; JHAWAR, N.; SAEDI, N. Tranexamic Acid for Melasma: Evaluating the Various Formulations. J Clin Aesthet Dermatol., v. 12, n. 8, p. E73-E74, 2019. 